\title{
El léxico y la enseñanza de la lengua: innovación y propuestas didácticas a partir del análisis de un método de enseñanza de español
}

\author{
Lexicon and Spanish Language Teaching: Innovation and Didactic Proposals \\ Based on the Analysis of a Spanish Language Teaching Method
}

Teresa Cervera Mata*

Recibido: 10 de agosto de 2012

Aceptado: 27 de septiembre de 2012

\section{Resumen}

La actual didáctica del léxico en enseñanza secundaria no está planificada ni es valorada, a pesar de la importancia del vocabulario en el aprendizaje de la lengua materna. Mediante el análisis de la metodología de un manual de enseñanza de lengua castellana y literatura en educación secundaria conoceremos las ventajas del sistema y los aspectos que se pueden revisar, para a continuación realizar propuestas didácticas con el fin de mejorar la adquisición del léxico por parte del alumnado. Basándonos en el enfoque comunicativo y en las deficiencias encontradas en el manual analizado, como la existencia de actividades tradicionales y de corte estructuralista, expondremos la necesidad de un cambio en la enseñanza del léxico, que debe ser planificada y contextualizada.

Palabras clave: léxico, metodología, enfoque comunicativo, semántica.

\section{Abstract}

In spite of the important role that lexicon plays in the process of mother tongue learning, lexicon didactics is currently unplanned and barely appreciated in the context of secondary education in Spain. This research, based on the analysis of a Spanish Language and Literature textbook and its methodology, intends to identify the advantages and weaknesses of this method in order to suggest didactic proposals which will help the students learn vocabulary. Taking into account the communicative perspective and the deficiencies found in the textbook that has been analyzed, as well as the existence of traditional activities with a structural approach, we will present the need of change in the way the vocabulary has been taught, one that must be planned and set in context.

Key words: lexicon, methodology, communicative approach, semantics.

* Actualmente es docente del Instituto de Educación Secundaria Río Andarax. Almería. España. Licenciada en Filología Hispánica, Universidad De Granada (2008). Máster universitario en Estudios Superiores de Lengua Española: Investigación y Aplicaciones. Universidad de Granada (2010).Correo electrónico: t.c.mata@gmail.com 


\section{Introducción}

\section{Principios pedagógicos para la enseñanza del léxico}

El objetivo del proceso de enseñanza-aprendizaje de una lengua es que el alumnado desarrolle de forma satisfactoria su competencia comunicativa. Esto, como afirma Gómez Molina (1997), “refleja cómo un hablante no solo posee un conocimiento de la lengua sino también una capacidad para utilizar o ejecutar dicho conocimiento cuando participa en una comunicación real” (p. 69).

Dentro de esta competencia comunicativa vamos a centrarnos en el campo de la enseñanza del léxico, cuyo fin es otorgarle al alumno el vocabulario necesario para convertirse en un hablante competente: "La enseñanza del léxico tiene como finalidad que las unidades léxicas pasen a la competencia comunicativa del individuo, dado el valor del vocabulario como elemento estructurador del pensamiento y su necesidad para la interacción social" (Gómez Molina, 1997, p. 71). Para ello investigamos, desde un punto de vista pedagógico, qué léxico, cuánto y cómo se puede aprender.

Es necesario destacar que actualmente no existe la planificación en el estudio del léxico. Esto puede deberse a varios motivos: la magnitud de la empresa y la valoración por parte de las diferentes corrientes metodológicas que privilegian otros aspectos del funcionamiento lingüístico. El léxico se incluye en las programaciones de secundaria y en los libros de texto de forma casi azarosa o al servicio del estudio de otros contenidos curriculares.

La importancia del léxico en el desarrollo de la competencia comunicativa no parece motivo suficiente para realizar una programación de este contenido, como lo vemos en manuales de enseñanza de español para extranjeros, en los que se programa el estudio de diferentes campos léxicos en función de las necesidades del alumnado, de los elementos más cercanos de su contexto o de sus expectativas futuras.

Ante esta situación es imprescindible preguntarnos si es posible una planificación del aprendizaje léxico. Antes de responder a esta cuestión, es necesario tener en cuenta los siguientes factores:

1. El Diccionario de la Real Academia ofrece 80000 palabras, de las cuales solo se enseñan las más utilizadas.

2. Existe un número relativamente pequeño de palabras que aparecen con mucha frecuencia, a tal punto que las 4000 palabras más frecuentes de una lengua abarcan el 97,5\% de un texto (Santiago, 2000, p. 155).

3. Es necesario que nos planteemos el contexto de aprendizaje: escolar o natural (el que afecta toda la vida).

4. Debemos situarnos en la legislación actual para los diferentes niveles educativos.

Para llevar a cabo esa planificación se debe partir del nivel de competencia que tienen los alumnos y ser conscientes de que es una parte del conocimiento lingüístico, por lo que se ha de estudiar de forma contextualizada. Sin embargo, como docentes nos encontramos con las siguientes dificultades en el estudio del léxico:

1. La ingente cantidad de términos.

2. La imposibilidad de estudiarlo sin el contexto.

3. La importancia tanto de la comprensión como de la producción.

4. La diferencia existente entre los términos.

Tras analizar la complejidad que presenta nuestro objeto de estudio nos planteamos la siguiente pre- 
gunta: ¿Qué léxico debemos enseñar? Para poder contestar, debemos conocer las diferencias existentes entre:

1. Lexema: unidad teórica del funcionamiento del nivel léxico del lenguaje.

2. Vocabulario: la realización concreta en un individuo.

Y entre:

1. Léxico general: el que está a disposición de todos los individuos.

2. Léxico disponible: los vocablos que el sujeto conoce pero que tienen una frecuencia muy baja.

Por último, hay que diferenciar entre:

1. Léxico fundamental: la suma del léxico general y el léxico disponible.

2. Léxico común: las palabras comunes a todos los idiolectos.

Es preciso señalar que todas las palabras no tienen la misma importancia funcional debido a la existencia de las diferencias diastráticas, diafásicas y diatópicas. Por lo tanto, la respuesta más concreta y directa a la pregunta planteada es "el léxico más frecuente". Sin embargo, nos pueden surgir dudas acerca de la enseñanza de los tecnicismos, de los vulgarismos o de los neologismos. Aun así, podemos ofrecer una conclusión inicial afirmando que enseñaremos un léxico determinado en función de la situación y la intencionalidad comunicativa.

\section{Métodos pedagógicos empleados en la enseñanza del léxico}

Los cambios que se han producido en la enseñanza hacen necesaria una transformación en la metodología. Una enseñanza memorística y teórica ha dado paso a una metodología activa y de participación.
Este cambio está relacionado con modificaciones en la descripción teórica sobre el funcionamiento del lenguaje, es decir, nace de las investigaciones en los diferentes campos teóricos.

Los avances en las investigaciones lingüísticas mejoran las propuestas que se ofrecen para su enseñanza y aprendizaje. Entre estas, coexisten planteamientos tradicionales con otros innovadores. Podemos distinguir entre dos métodos, fundamentalmente, el estructuralista y el comunicativo:

1. Método estructuralista: basado en el principio de sustitución. Ha generado ejercicios que se sustentan en este criterio, formales, mecanicistas y desarrollados al margen del uso real de la lengua.

2. Método comunicativo: basado en el principio de comunicación. Se quiere acercar al alumno al uso real de la lengua, por lo que se ofrecen textos reales, hecho que deja de lado todo principio de selección del vocabulario.

El alumno ha de trabajar con realia y practicar en situaciones comunicativas reales (o simuladas en el aula). Las nuevas unidades léxicas deben presentarse contextualizadas: textos, entre los que se incluyen también los literarios puesto que debe integrarse la enseñanza de la lengua y la literatura. (Gómez Molina, 1997 p. 72)

Se ha puesto de manifiesto que las unidades léxicas no se almacenan al azar o de manera aislada en la mente, sino que están organizadas en redes interconectadas, por lo que también se emplean mapas conceptuales para su aprendizaje. En el método comunicativo hay que destacar el aprendizaje por tareas, en el que las teorías pedagógicas han desarrollado el principio de aprender a aprender. Para que este enfoque comunicativo se aplique con éxito es imprescindible que "el aprendizaje se desarrolle al unísono en diversos planos: visual, auditivo, semántico, pragmático y motórico" (Gómez Molina, 1997, p. 72). 
Investigaciones como la de Rueda Bernao (1994), en "Análisis de cuatro manuales para la enseñanza del español, lengua extranjera", ofrecen un precedente en nuestra investigación, aunque centrado en el análisis de la enseñanza del español como lengua extranjera. En aquel estudio, cuatro manuales de enseñanza de español para extranjeros fueron examinados tomando como punto de partida unos criterios comunicativos, para así comparar las diferentes líneas metodológicas.

Tras estas reflexiones, sin embargo, podemos afirmar que, tal y como expondremos en el tercer apartado de este estudio, los actuales manuales de lengua castellana y literatura no presentan una planificación de la enseñanza del léxico. La metodología planteada para su desarrollo responde más a un modelo estructuralista que comunicativo. Esta es una corriente habitual en las programaciones didácticas que, aunque introducen paulatinamente el modelo comunicativo y el enfoque por tareas, estos no se aplican a todas las actividades ni a todos los bloques de contenidos. Quizás sea el estudio del léxico el más afectado por esta problemática, debido al enfoque tradicional de su enseñanza a través de actividades de repetición, de cumplimentación de espacios o de elaboración de frases descontextualizadas.

Ante la situación que estamos planteando, hay que ser conscientes, como afirma Reyes Díaz (1999), de que el éxito "dependerá no solo de la capacidad y de la motivación que reciba el estudiante y de la dificultad o del nivel de abstracción del vocablo, sino del método de contacto utilizado" (p. 91).

Para ello, tras el análisis del manual de enseñanza de español como lengua materna que realizaremos en el tercer apartado, formularemos una serie de propuestas didácticas que pensamos que se nutren del espíritu comunicativo y que persiguen el aprendizaje del léxico por medio de actividades contextualizadas y productivas. Consideramos de notable importancia la investigación en este campo pues, tras el ingente esfuerzo de investigadores y miembros de la comunidad educativa por transformar una metodología conservadora y mecanicista, es necesario aplicar este espíritu de cambio a todos los bloques de contenidos que afectan el currículo de la enseñanza de la lengua, y en especial, a uno de gran relevancia como la palabra, elemento básico en la elaboración del lenguaje.

\section{Objetivos del trabajo}

Con este trabajo pretendemos realizar el análisis de un método de enseñanza de español como lengua materna, dirigido a alumnos de cuarto curso de educación secundaria, en el ámbito de la enseñanza del léxico. Tras el análisis de las diferentes unidades didácticas que lo componen y los apartados dedicados al vocabulario, realizaremos propuestas didácticas que en nuestra opinión mejoren el método en este aspecto. Por último, intentaremos extraer conclusiones que puedan contribuir a optimizar el método de enseñanza del léxico en el aprendizaje de la lengua materna.

\section{Análisis de un método de enseñanza de español como lengua materna}

\section{Justificación de la propuesta}

El libro que hemos seleccionado para el análisis está dirigido a alumnos de cuarto curso de educación secundaria obligatoria (ESO). Fue publicado en el 2006 por la editorial Anaya y sus autores son Fernando Lázaro Carreter, Rosa Llupart, Joaquín Mindán y Eduardo Gimeno.

Hay diversos motivos que justifican esta elección. En primer lugar, el método contiene un apartado reservado para la enseñanza del léxico que se titula "Enriquece tu vocabulario". Creemos que es interesante estudiar un método que disponga de un espacio reservado para la enseñanza de esta categoría lingüística, pues refleja la importancia que a ello le 
dan sus autores. También estudiaremos cómo se enseña de forma transversal en el resto de las secciones que dividen cada unidad didáctica. Otro motivo es que no presenta división entre las diferentes áreas de estudio: lengua, comunicación y literatura. En cada unidad didáctica podemos observar cómo se aúnan y complementan estos tres apartados. Pensamos que la división podría afectar la enseñanza del léxico porque, como sabemos, este no se puede enseñar de forma aislada sino contextualizada.

\section{Análisis de la enseñanza del léxico}

En primer lugar, vamos a realizar un análisis de la estructura del método elegido para estudiar su composición y comprobar en qué ocasiones se enseña el léxico.

El método está dividido en dieciséis unidades didácticas que, como hemos dicho, aúnan las áreas de comunicación, lengua y literatura. Comprende las siguientes secciones:

\section{Lectura}

Quince de las dieciséis unidades inician con un texto de carácter literario. Sin embargo, en la unidad 14 encontramos un artículo periodístico de La Vanguardia. Al ser textos que forman parte de una obra publicada, el método que analizamos está dando su primer paso hacia un enfoque comunicativo, pues está empleando textos reales para el estudio lingüístico.

En segundo lugar, se plantea un apartado titulado "Comprensión del texto", en el que el alumno desarrolla su comprensión lectora a través de una serie de preguntas. Por lo general son cuestiones que intentan hacer reflexionar acerca del sentido de la lectura; sin embargo, en muchas ocasiones se utilizan estas preguntas para introducir al alumno en los contenidos teóricos que se desarrollarán más adelante.

Por último, se programa en esta introducción de la unidad didáctica una sección titulada "Enriquece tu vocabulario", que se nutre del fragmento analizado anteriormente. Es la parte dedicada a la enseñanza de términos y su objetivo es aumentar el caudal léxico del alumnado. Se compone de unos cuatro o cinco ejercicios, dependiendo de la unidad, y se tratan los siguientes aspectos:

Semántica: uno de los principales objetivos que se plantean es que el alumno conozca el significado de los diferentes términos y que sepa relacionarlos con la familia léxica a la que pertenecen. La forma de plantear estos ejercicios es muy diferente entre sí $y$, por lo tanto, también lo es el resultado que se puede obtener. Exponemos a continuación una serie de ejemplos:

1. ¿En qué se diferencian expiaría, que aparece al final del texto, y espiaría? Precisa el significado de cada uno (Carreter, Llupart, Mindán y Gimeno, 2006, p. 45).

En este ejercicio se plantean dos verbos en condicional con una sola diferencia fonética y se pide que se especifiquen sus significados para no confundir los términos. El estudio del léxico está contextualizado porque los términos se obtienen del texto, que en este caso es un fragmento de la obra Shakespeare cuenta... Sin embargo, el ejercicio es muy formal. Se busca únicamente la diferencia de significado.

2. Emancipado, explícitamente y efímera son cultismos, proceden del latín y del griego. Si lo necesitas, busca el significado de estas palabras en el diccionario y escribe una frase con cada una de ellas (Carreter et al., 2006, p. 27).

Este es uno de los ejercicios más tradicionales en el planteamiento; es también de los más comunes. Responde a una visión estructuralista y formal de la enseñanza de la lengua, pues no se plantea el aprendizaje de estos términos de una forma comunicativa. Es necesario animar al alumnado a buscar palabras en el diccionario, pues deben acostumbrarse a esta 
práctica, pero la forma de asimilar los conceptos que están aprendiendo quizás no sea la más adecuada. Escribir una frase con cada palabra, aunque intenta potenciar la creatividad, no pone en práctica el uso real de la lengua. No buscamos únicamente que el alumno conozca el significado del término sino que aprenda a emplearlo en su contexto porque, como afirma Gómez Molina (1997): “el dominio del léxico depende de las relaciones que el alumno sea capaz de establecer entre una unidad léxica y otras, tanto en su aspecto semántico-pragmático como en la capacidad combinatoria que esa unidad permita” (p. 75).

3. Escribe en tu cuaderno tres nombres del texto que hagan referencia al mundo de la caballería. (Carreter et al., 2006, p. 113).

Se pide que el alumno agrupe las palabras que pertenezcan a la familia léxica de la caballería. Este tipo de ejercicios es interesante pues constituye un primer acercamiento a las voces que se están estudiando; aunque no se conozca el significado concreto, saber a qué familia de palabras pertenece implica conocer una parte de su significado. El problema que se plantea en este caso es que posteriormente no se pide al alumno que siga estudiando esos términos o que los utilice en una práctica comunicativa, sino que se pasa a trabajar otras palabras diferentes.

4. ¿A qué campo semántico pertenecen las palabras bicloruro de mercurio, sulfúricas, solimán $y$ cinabrio? (Carreter et al., 2006, p. 64).

Encontramos un planteamiento contrario al anterior, en el que nos ofrecen las palabras y debemos nombrar el campo semántico. En esta ocasión sí parece oportuno no seguir trabajando con los términos, pues no pertenecen al acervo de palabras frecuentes y su conocimiento no es imprescindible para el alumnado. Para esta afirmación nos apoyamos en la opinión de Giammatteo, Albano, Trombetta y Ghío (2001):
El conocimiento del vocabulario tiene varias dimensiones. Acceder a los niveles superiores, cuando tienen que ver con áreas específicas, implica dominar estrategias que permitan partir del simple reconocimiento de los términos en un ámbito de uso determinado y ascender en su conocimiento, a través de la incorporación del fragmento de realidad al que la palabra hace referencia. [...] La instrucción debe así ir conduciendo a verdaderas estrategias de aprendizaje de base léxico-cognitivas. (p. 66)

5. Escribe las palabras del texto correspondientes al campo de la informática y averigua si son monosémicas o polisémicas. Escribe dos frases con distintos significados para cada una de las polisémicas. (Carreter et al., 2006, p. 215).

Exceptuando el último apartado del ejercicio que se podía haber planteado de forma más comunicativa, pensamos que es una buena actividad. No solo se pide una clasificación de los términos según el campo semántico al que pertenecen, sino también según sus significados. De esta manera, para averiguar este último aspecto, el alumno deberá conocer la semántica de las palabras que se estudian.

Dentro de este apartado podemos destacar el estudio de:

Sinónimos y antónimos: otra de las actividades más frecuentes es conocer los sinónimos y antónimos de una serie de palabras.

6. Escribe un sinónimo de cada una de las palabras subrayadas en las frases que hay a continuación: un buen hijo no emancipado/ indagues lo que pasó/ fueron una leyenda en su momento... (Carreter et al., 2006, p. 27).

Aquí observamos un planteamiento estructuralista, pues la sustitución de unos términos por otros es el centro de la actividad.

7. Busca un sinónimo de iracundo y otro de ruboroso (Carreter et al., 2006, p. 45). 
En esta ocasión, con la búsqueda del sinónimo se persigue que el alumno conozca el significado de la palabra sin dar una definición. Al igual que en el ejercicio anterior, una vez que se conocen los sinónimos se podría plantear una práctica en la que los emplearan en el uso real de la lengua.

8. Busca un sinónimo para cada uno de los siguientes términos: solución, acertijo, problema, tregua, juego (Carreter et al., 2006, p. 215).

De los ejercicios analizados, este es el más tradicional y formal. Ofrecer una lista de palabras sin contextualizar (aunque hayan sido extraídas de un fragmento leído anteriormente) es un método excesivamente mecánico y puede resultar tedioso para los alumnos.

Derivación: estudiar la derivación como proceso de formación de palabras es imprescindible para el conocimiento del español, y sobre todo del léxico. En ese sentido este método no ofrece un número elevado de ejercicios dedicados al conocimiento de los sufijos y de los prefijos que componen las palabras.

9. ¿Qué tipo de morfema hay en cafecito? ¿Qué significado aporta al lexema? (Carreter et al., 2006, p. 97).

El conocer los significados de los diferentes morfemas es necesario y ayuda a comprender en qué aspectos cambian las palabras cuando se les añaden. En este caso, el sufijo cito es de uso frecuente, por lo que su estudio es muy útil para el alumno.

10. El adverbio anteriormente deriva del adjetivo anterior. Escribe los adverbios acabados en mente de ágil, fácil, remoto, constante y útil (Carreter et al., 2006, p. 165).

Es un ejercicio muy mecánico y simple que no ofrece más posibilidades que las evidentes y no exige al alumno que potencie su creatividad.
11. ¿De qué palabra deriva el adjetivo desdeñosa? Escribe una frase con ella (Carreter et al., 2006, p. 45).

En esta ocasión no se estudian los sufijos pero se pide que se descarten para obtener la raíz del término. Se está haciendo hincapié en el carácter derivativo de la lengua. Como ya hemos dicho, pedir la redacción de una frase no acerca al alumno al uso real de la lengua.

Dentro de este apartado podemos incluir aquellos ejercicios en los que se pide al alumno que diferencie entre palabras compuestas y derivadas, o si el término que se estudia pertenece a una u otra categoría:

12. La palabra enhorabuena está formada por varios monemas. Indica si es una palabra derivada o compuesta y explica su significado (Carreter et al., 2006, p. 265).

13. Encuentra en el texto cuatro palabras derivadas, explica sus respectivos significados en tu cuaderno y construye una frase con cada una de ellas (Carreter et al., 2006, p. 64).

Fraseología: la sección "Enriquece tu vocabulario" no se limita únicamente a la enseñanza de palabras, sino que también plantea ejercicios para aprender el significado de ciertas colocaciones, locuciones o enunciados fraseológicos, como los siguientes:

14. En los versos 87 y 88 se dice que los escribanos dieron fe a la escena. ¿Qué quiere decir esta expresión? ¿En qué ámbito se usa actualmente? (Carreter et al., 2006, p. 130). Explica el significado de las expresiones hacer honor a, hacer los honores $y$ en honor de (Carreter et al., 2006, p. 113).

15. Explica en qué consiste cada uno de los siguientes trabajos: trabajo intelectual, trabajo burocrático, trabajo artesanal, trabajo técnico, trabajo a 
destajo, trabajo a tanto alzado (Carreter et al., 2006, p. 231).

El problema que hemos encontrado en estos ejercicios es que únicamente se persigue que el alumno conozca el significado de las diferentes expresiones, pero no se profundiza en su estudio ni se pide que se apliquen en contextos reales, hecho que contribuiría a su aprendizaje.

\section{Comunicación}

La unidad didáctica continúa con este apartado en el que se estudian los diferentes tipos de textos (expositivos, argumentativos, jurídico-administrativos, etc.) y los medios de comunicación audiovisual (prensa, radio y televisión). En esta sección no se trabaja el léxico.

\section{Gramática}

En este apartado se trata la situación actual del español, sus dialectos meridionales y el español de América, así como conceptos relacionados con la gramática del texto y las estructuras de las oraciones simple y compuesta. Está dividida en dos secciones: "El español actual" y "Norma y uso". En la primera, en algunos casos los temas están dedicados a la enseñanza del léxico y en la segunda se plantean actividades relacionadas con la explicación inicial o simplemente con aspectos normativos. Estos son algunos de los temas tratados relacionados con la enseñanza del léxico:

1. El léxico del español. Pertenece a la primera unidad didáctica. Aquí nos encontramos con una extensa explicación acerca del origen del léxico del español. En ella se exponen las diferencias entre voces patrimoniales, cultismos, dobletes y préstamos (dentro de los cuales encontramos germanismos, arabismos, galicismos, italianismos, lusismos, catalanismos, vasquismos y galleguismos). La explicación de los diversos orí- genes va acompañada de una enumeración de palabras como ejemplo. Es acertado comenzar el método explicando el origen de las voces que componen el español, pues para estudiar una materia es necesario conocer dónde comienza a formarse el objeto de estudio. En el apartado de "Norma y uso" se desarrollan actividades muy interesantes, aunque con algunas carencias. En la mayor parte de ellas se pide que se busque el significado de determinados términos pero no se pone en práctica su uso, sino que se propone construir una frase o un texto con ellos o que se rellenen espacios vacíos. Volvemos a ver el método estructuralista basado en la sustitución.

2. La influencia del inglés y los neologismos. Esta explicación continúa con la de la unidad anterior y se centra en las palabras que proceden del inglés y en las de nueva incorporación. Es un tema interesante pues se trabajan términos muy actuales con los que los alumnos suelen estar relacionados. También se estudian las siglas y los acrónimos, y se dan como ejemplos los que usan con más frecuencia. Los ejercicios que se proponen en el apartado de "Norma y uso" siguen la estructura de los de la sección "Enriquece tu vocabulario". En muchas ocasiones son ejercicios de sustitución, para buscar en el diccionario, inventar frases con los diferentes términos o distinguir entre palabras derivadas o compuestas. En esta unidad también se pide el significado de una serie de siglas.

3. El español de América: el léxico. Al igual que en la primera unidad didáctica, se procede a una clasificación del léxico según su origen, pero en este caso del léxico americano. Este método dedica dos unidades didácticas a tratar el tema del español de América. Consideramos que es un espacio excesivo si tenemos en cuenta que uno de los objetivos que nos planteamos en la 
enseñanza del español, y más concretamente en la del léxico, es la de enseñar los vocablos más frecuentes. Aunque es necesario que los alumnos conozcan algunas de las diferencias que existen entre la variante peninsular y la americana, no es imprescindible una profundización en estos aspectos. En muchos casos los métodos de enseñanza los incluyen por ser políticamente correctos y no es este el objetivo que debería primar en su elaboración.

\section{Uso de la lengua}

Este apartado está compuesto por dos secciones: “Ortografía” y “Técnicas de trabajo”. En el primero se trabajan los signos prosódicos, la tilde y el uso de algunas letras que pueden llevar a confusión. En el segundo se ofrecen varias estrategias para la búsqueda y presentación de textos en los nuevos soportes. Como es lógico, el primero es el que está más relacionado con el uso del vocabulario. Sin embargo, los criterios que se tienen en cuenta para elegir las palabras sobre las que se trabaja no siguen unos principios relacionados con la enseñanza del léxico, sino principios ortográficos. Aun así, se proponen algunas actividades que pueden ser interesantes y que pueden responder al objetivo de aumentar el caudal léxico del alumnado:

1. Forma los plurales de estas abreviaturas y di qué significa cada una: A., Rte, Fe, s., pág., Sr., Dir., km, ms., fca., n. ${ }^{\circ}$ (Carreter et al., 2006, p. 33).

2. Consulta en el diccionario de la Real Academia Española si las siguientes siglas son consideradas acrónimos o no: pyme, NIF, Talgo, CD-ROM, TAC, PC, VIP (Carreter et al., 2006, p. 88).

3. Indica cuál es el gentilicio de cada uno de los siguientes lugares: Eslavia, Suecia, Praga, Londres, Perú, Suiza, Costa Rica, Dinamarca, Albania, Bélgica, Marruecos, Berlín, Chad, Moscú,
Andorra, París, Afganistán, Argel (Carreter et al., 2006, p. 121).

En estas actividades vemos una carencia común: el uso de listas de palabras descontextualizadas. Para aprender léxico u ortografía (lo primero implica lo segundo), es necesario contextualizar el aprendizaje $\mathrm{y}$ ofrecer ejercicios que fomenten la creatividad del alumno. Realizar una tarea en la que sea necesario el uso de los términos conllevaría unos resultados más positivos, así como hacer un uso real de estos. Sin embargo, vemos que en la segunda actividad se ha usado un elemento muy útil que es el de la consulta del Diccionario de la Real Academia, no solo para obtener el significado, sino también para conocer si son acrónimos o no. Es muy importante enseñar a manejar los diferentes diccionarios y las posibilidades que nos ofrecen, más allá de los significados.

\section{Literatura}

Aquí se plantea un tratamiento cronológico de la literatura desde comienzos del siglo XIX hasta la actualidad. Aunque en menor medida, encontramos también actividades relacionadas con el ámbito léxico, y en este caso sí son palabras contextualizadas, pues suelen analizarse partiendo de textos literarios del periodo que se está estudiando:

1. Anota todas las palabras del texto relacionadas con el mundo romántico (Carreter et al., 2006, p. 39).

En esta actividad se le pide al alumno que seleccione las palabras que pertenezcan al campo semántico del romanticismo. Se supone que ya se conocen, pero se busca ampliar la información acerca del movimiento literario, para lo cual se aúnan las palabras que a ello se refieren.

1. ¿Qué nombre reciben, en narrativa, los saltos al pasado? (Carreter et al., 2006, p. 93). 
2. En este caso se da el significado y se busca el término que está en el texto que se ha leído anteriormente.

3. Localiza algún ejemplo de desajuste léxico (mezcla de registros lingüísticos) en el texto (Carreter et al., 2006, p. 161).

4. Este ejercicio es muy interesante para los alumnos de educación secundaria, pues saber que no se pueden emplear todos los términos por igual, sino que se debe hacer una selección en función del registro, será muy útil para ellos al poner en práctica el uso de la lengua.

\section{Lectura y comentario}

La unidad se cierra con una nueva lectura, para que los alumnos realicen un breve y básico comentario de texto guiado a través de unas preguntas.

1. Anota las palabras que te parezcan cultas o exóticas y que dan belleza al texto (Carreter et al., 2006, p. 111).

Este ejercicio pide una valoración personal acerca de los vocablos por parte del alumno, al igual que la siguiente.

2. Anota todas las expresiones evocadoras referidas a un tiempo pasado que aparezcan en el texto (Carreter et al., 2006, p. 163).

Se pide que se clasifiquen los términos según el campo semántico al que se refieran.

3. Para Salinas el amor es perfección, conocimiento, elevación. Señala las expresiones que indican esa ascensión como sinónimo de perfección y enriquecimiento (Carreter et al., 2006, p. 213).

En este ejercicio se vuelve a incidir en el aspecto semántico, en este caso a través de sinónimos.

\section{Conclusiones del análisis del método}

1. La sección dedicada con exclusividad al estudio del léxico es un aspecto favorable del método. Sin embargo, no observamos que exista planificación. En algunos casos se seleccionan los textos y las palabras que se van a estudiar en función de los aspectos teóricos (neologismos, derivación, etc.), pero esto no ocurre siempre.

2. Podemos encontrar actividades dedicadas a la enseñanza del léxico a lo largo de todas las unidades didácticas, hecho que favorece el estudio de los textos que se están trabajando.

3. Casi todos los ejercicios pertenecen al método estructuralista. Aunque es positivo que la mayoría de las voces que se estudien estén contextualizadas porque proceden de una lectura, las actividades que se plantean carecen de creatividad y son meros ejercicios de sustitución o de búsqueda de significado.

4. No encontramos propuestas de trabajo por parejas. Todos los ejercicios se preparan para ser realizados de forma individual y ser posteriormente corregidos por el profesor o en una sesión plenaria.

5. El método carece de actividades lúdicas y de ejercicios introductorios que sirvan de motivación al estudio del léxico. También carece de las nuevas estrategias de enseñanza léxica basadas en el uso de constelaciones y en la dramatización.

6. Las únicas aportaciones reales para la contextualización del léxico son textos literarios, pero se dejan de lado otras posibilidades como revistas, periódicos, películas o canciones.

7. La puesta en práctica del léxico aprendido no se inserta dentro de un uso real de la lengua, 
sino que se ciñe a la creación de frases independientes y sin contexto.

8. Las actividades que se proponen no tienen repercusión fuera del aula.

9. No existen métodos para evaluar las estrategias empleadas ni los resultados obtenidos.

\section{Propuesta de enseñanza del léxico y ejercicios concretos]}

A continuación se exponen las propuestas didácticas para la enseñanza del léxico. Se trata de una serie de actividades que pensamos que pueden resultar motivadoras, ayudar al desarrollo de la creatividad $y$, sobre todo, que aumentan el caudal léxico del alumnado al que va dirigido. Como el método que hemos analizado anteriormente está pensado para alumnos de cuarto de ESO, creemos oportuno realizar las propuestas didácticas adaptadas a este nivel de estudios. Se basan en el enfoque comunicativo e intentan llevar a la práctica algunas de las estrategias didácticas planteadas por Moreno (2002). Él propone seis estrategias léxicas: convencionales, lúdicas, contextualizadas, realias, constelaciones y dramatizaciones. Nosotros aplicamos distintas estrategias en una misma actividad o tarea en función de los objetivos propuestos.

\section{Nuevos profesores}

Objetivos:

1. Aumentar el caudal léxico de los alumnos.

2. Fomentar su creatividad en el ámbito de la comunicación escrita.

3. Aprender a utilizar los diccionarios manuales y digitales.

4. Fomentar el aprendizaje autónomo.
5. Incentivar al alumno a través de la preparación de actividades para los demás.

Para llevar a cabo el siguiente ejercicio, realizaremos una constelación.

Las constelaciones léxicas son un recurso para explorar y extraer vocabulario de manera cooperativa, inductiva y dinámica. A partir de esta técnica y con las palabras resultantes podemos emprender otras tareas lingüísticas. [ ] El concepto fundamental es que todos los vocablos entablan múltiples vinculaciones lingüísticas; las más importantes son las cinco siguientes: sinonimia, antonimia, derivación, composición y asociación (fónica y semántica). Se tratará por tanto, de mostrar las conexiones o redes léxicas realizada a partir de una palabra. (Moreno, 2002, p. 83)

Seguiremos los pasos que detallamos a continuación:

1. Se divide al alumnado en grupos de cuatro o cinco integrantes y a cada uno se le entrega uno de los vocablos generadores: colegio, abogado, tenis, jardín y gimnasio.

2. Los alumnos realizan la constelación que tendrá el tamaño de un folio. Los términos se irán anotando según vayan surgiendo a partir del vocablo generador.

3. Cuando se finalicen las constelaciones cada grupo selecciona entre cinco y diez vocablos pertenecientes al vocabulario pasivo y potencial.

4. A continuación los alumnos realizan en grupos las siguientes tareas relacionadas con esos términos:

a. ¿Cuál es el significado de esta palabra? Si no conocéis el término buscadlo en el diccionario.

b. Redactad una historia con dos finales alternativos en la que encontremos esos términos y después contádsela al resto de los compañeros de la clase. 
c. Preparad dos ejercicios para que los compañeros de otros grupos puedan trabajar estos términos.

5. Los alumnos, en los mismos grupos a los que pertenecían anteriormente, realizan las actividades propuestas por sus compañeros.

6. Se evalúa la sesión a través de la corrección de las actividades en sesión plenaria y de un coloquio general.

\section{Yahora... escritores}

Objetivos:

1. Aumentar el caudal léxico del alumnado.

2. Desarrollar la competencia escrita.

3. Fomentar la creatividad.

4. Fomentar el trabajo en grupo.

a.En primer lugar se deberá realizar dos lecturas del siguiente texto, la primera individual y la segunda en voz alta para todos.

El Cementerio de los Libros Olvidados

Todavía recuerdo aquel amanecer en que mi padre me llevó por primera vez a visitar el Cementerio de los Libros Olvidados. Desgranaban los primeros días del verano de 1945 y caminábamos por las calles de una Barcelona atrapada bajo cielos de ceniza y un sol de vapor que se derramaba sobre la Rambla de Santa Mónica en una guirnalda de cobre líquido. -Daniel, lo que vas a ver hoy no se lo puedes contar a nadie - advirtió mi padre-. Ni a tu amigo Tomás. A nadie. - ¿Ni siquiera a mamá? - inquirí yo, a media voz. Mi padre suspiró, amparado en aquella sonrisa triste que le perseguía como una sombra por la vida. -Claro que sí - respondió cabizbajo-. Con ella no tenemos secretos. A ella puedes contárselo todo. Poco después de la guerra civil, un brote de cólera se había llevado a mi madre. La enterramos en Montjuïc el día de mi cuarto cumpleaños. Sólo recuerdo que llovió todo el día y toda la noche, y que cuando le pregunté a mi padre si el cielo lloraba le faltó la voz para responderme. Seis años después, la ausencia de mi madre era para mí todavía un espejismo, un silencio a gritos que aún no había aprendido a acallar con palabras. Mi padre y yo vivíamos en un pequeño piso de la calle Santa Ana, junto a la plaza de la iglesia. El piso estaba situado justo encima de la librería especializada en ediciones de coleccionista y libros usados heredada de mi abuelo, un bazar encantado que mi padre confiaba en que algún día pasaría a mis manos. Me crié entre libros, haciendo amigos invisibles en páginas que se deshacían en polvo y cuyo olor aún conservo en las manos. De niño aprendí a conciliar el sueño mientras le explicaba a mi madre en la penumbra de mi habitación las incidencias de la jornada, mis andanzas en el colegio, lo que había aprendido aquel día... No podía oír su voz o sentir su tacto, pero su luz y su calor ardían en cada rincón de aquella casa y yo, con la fe de los que todavía pueden contar sus años con los dedos de las manos, creía que si cerraba los ojos y le hablaba, ella podría oírme desde donde estuviese. A veces, mi padre me escuchaba desde el comedor $y$ lloraba a escondidas. Recuerdo que aquel alba de junio me desperté gritando. El corazón me batía en el pecho como si el alma quisiera abrirse camino y echar a correr escaleras abajo. Mi padre acudió azorado a mi habitación y me sostuvo en sus brazos, intentando calmarme. No puedo acordarme de su cara. No puedo acordarme de la cara de mamá - murmuré sin aliento. Mi padre me abrazó con fuerza. - No te preocupes, Daniel. Yo me acordaré por los dos. Nos miramos en la penumbra, buscando palabras que no existían. Aquella fue la primera vez en que me di cuenta de que mi padre envejecía y de que sus ojos, ojos de niebla y de pérdida, siempre miraban atrás. (Ruiz, 2004, p. 2).

b. A continuación los alumnos, por parejas, realizan las siguientes actividades:

5. Seleccionad del texto aquellas palabras que desconocéis y las que conocéis pero no utilizáis frecuentemente.

6. Buscad el significado de las palabras que habéis seleccionado anteriormente.

7. Redactad un final para la historia que habéis comenzado a leer: ¿qué le va a enseñar su padre 
a Daniel? ¿Qué sitio es y cómo de importante? ¿Por qué es un secreto? Para elaborar el final debéis emplear las palabras que habéis seleccionado anteriormente.

8. Leed vuestro final al resto de la clase.

9. Reescribid las frases anteriores tal y como las habríais pronunciado vosotros:

- Nos miramos en la penumbra.

- Mi padre acudió azorado a mi habitación $\mathrm{y}$ me sostuvo en sus brazos.

- - ¿Ni siquiera a mamá? -inquirí yo, a media voz.

- De niño aprendí a conciliar el sueño mientras le explicaba a mi madre en la penumbra de mi habitación las incidencias de la jornada, mis andanzas en el colegio

\section{Diccionario en Internet}

\section{Objetivos}

1. Aprender a utilizar los diccionarios digitales.

2. Aprender a utilizar las nuevas tecnologías de una forma útil y responsable.

El momento en el que los alumnos tengan dudas acerca de alguno de los términos que se trabajan en clase, podemos utilizar el Diccionario de la Real Academia en versión digital a través de Internet, que es una herramienta muy útil (www.rae.es). Si nos encontramos en un centro de tecnologías de la información y la comunicación (TIC) esta actividad puede ser muy provechosa:

1. Consultar el significado del término.

2. Si es un verbo, enseñar al alumno cómo debe hacer para consultar la conjugación.

3. Si es una duda morfológica o sintáctica en el apartado de consultas lingüísticas, el alumno puede enviar su pregunta a la Academia por correo electrónico y el personal encargado le contestará en el plazo de una semana.

4. Otra vía para solucionar las dudas que se nos planteen en clase es la consulta del Diccionario panhispánico de dudas, también de la Real Academia Española, a través de Internet (www. rae.es).

\section{¿Ensayamos?}

\section{Objetivos}

1. Aumentar el caudal léxico del alumnado.

2. Mejorar la competencia oral.

3. Fomentar la creatividad.

4. Ayudar a la desinhibición.

Esta actividad es una dramatización, que Moreno (2002) define así:

Las técnicas de simulación son un buen recurso para la enseñanza y el aprendizaje de idiomas; sin embargo, en nuestro país y por motivos diversos, este tipo de herramientas apenas han sido aplicadas en la tradición escolar de la lengua materna. [...] Entre las virtualidades pedagógicas de la dramatización destacamos sobre todo el carácter globalizador del lenguaje (aúna la expresión verbal y no verbal) y su multidisciplinariedad (expresión corporal, lingüística, plástica y ritmo-musical); además propicia procesos de integración entre los componentes por los ingredientes cooperativos lúdicos de esta metodología. (p. 84)

Los pasos que seguiremos serán los siguientes:

1. Dividiremos la clase en tres grupos.

2. Cada uno de estos grupos llevará a cabo una interpretación diferente, basada en un ambiente distinto y con una serie de personajes tipo.

3. A los diferentes grupos se les adjudicará un vocabulario que deberán aplicar en las situaciones que representen. 
a. Grupo A: la gestoría. Personajes: el abogado, el secretario, tres clientes y el cartero. Léxico: instancia, declaración de hacienda, impuestos, hipoteca, IVA

b. Grupo B: el hospital. Personajes: el médico, el enfermero, el auxiliar de clínica, el radiólogo y tres pacientes. Vocabulario: fisura, radiografía, ecografía, análisis, enfermedades cuya terminación sea itis (amigdalitis, faringitis, apendicitis...), sutura, férula

c. Grupo C: el vivero. Personajes: dos jardineros y tres clientes. Vocabulario: trasplantar, abonar, agronomía, regadío...

4. Los alumnos realizan las representaciones para el resto de la clase.

\section{En el periódico}

\section{Objetivos}

1. Aumentar el caudal léxico de los alumnos.

2. Fomentar su creatividad.

3. Conocer la estructura interna de un periódico.

4. Mejorar la capacidad de redacción.

La estrategia que emplearemos para conseguir nuestros objetivos será crear un periódico que, una vez terminado, podremos distribuir por el centro escolar. Para su realización dividiremos la clase en tantos grupos como secciones queremos que constituyan nuestra publicación: portada, editorial, noticias nacionales, internacionales, deportes, entretenimiento

Los alumnos de cada grupo, mediante la búsqueda en periódicos digitales y en papel, elegirán algunas de las noticias más destacadas para cada sección. Una vez seleccionados los artículos serán trabajados de la siguiente manera:
1. Elección del vocabulario desconocido y del conocido pero menos empleado.

a. Estudio de este vocabulario (significado, campo semántico )

2. Redacción propia de las noticias empleando la mayor parte del vocabulario analizado.

Una vez escritos los artículos la clase entera procederá a la maquetación del periódico a través de sugerencias de presentación. Por último, se distribuirá al resto de alumnos del centro escolar. Para que resulte más atractivo, se pueden incluir secciones locales o referentes al centro de estudios.

\section{Conclusiones}

A continuación presentamos brevemente algunas de las conclusiones a las que hemos llegado tras el análisis del método de enseñanza de la lengua y de la bibliografía comentada:

1. La enseñanza del léxico es un ámbito demasiado importante en el estudio de una lengua como para pasarlo por alto. Aumentar el léxico disponible y potencial de un alumno supone mejorías en las competencias oral y escrita. La expresión lingüística será mucho más rica y fluida cuantos más términos se conozcan y se sepan utilizar. No solo los aspectos morfológicos, sintácticos y discursivos ayudarán a los alumnos a desarrollar estas capacidades; el ámbito léxico es indispensable.

2. Es necesario incluir en los métodos de enseñanza de la lengua un apartado dedicado a la enseñanza del léxico. A pesar de que es positivo encontrar actividades con este fin a lo largo de las diferentes unidades didácticas, es un ámbito de tanta importancia que exige una atención especial, o por lo menos la misma que el resto de ámbitos lingüísticos, hecho que no siempre ocurre. 
3. Es necesaria una planificación de esta enseñanza. El léxico no debe proponerse en el método como consecuencia de los textos seleccionados, sino a la inversa, proponer las fuentes en función del vocabulario que queremos enseñar.

4. El método que hemos analizado presenta las siguientes potencialidades:

a. El léxico se enseña de forma contextualizada, a partir de textos literarios.

b. Existe un apartado específico para la enseñanza del léxico.

c. A lo largo de las unidades didácticas nos encontramos con ejercicios dedicados a este fin, aunque sea en apartados diferentes.

4. Sin embargo, también podemos destacar las siguientes carencias:

a. Las actividades son muy mecánicas y no fomentan la creatividad.

b. No existen ejercicios de motivación que animen al alumno al comienzo de la actividad.

c. La mayor parte de los ejercicios se basan en la búsqueda de significados, sinónimos o antónimos, y en el conocimiento de las posibles derivaciones y de los elementos necesarios para ello.

d. La puesta en práctica del vocabulario que se trabaja se limita a su aplicación en oraciones inventadas por el alumno y no se fomenta su inclusión en el uso real de la lengua.

e. No se fomenta el trabajo en grupo.

f. No se anima a la realización de grandes tareas que puedan exponerse en el centro educativo.
5. Tras un resumen de las conclusiones a las que llegamos después del análisis, podemos realizar la siguiente afirmación: el método analizado, a pesar de tener características propias del enfoque comunicativo como la contextualización, sigue una línea de trabajo estructuralista basada en el método de la sustitución.

6. El presente estudio asume las siguientes limitaciones que deberán ser trabajadas en una investigación posterior:

a. El diseño de una programación didáctica tomando como eje central la enseñanza del léxico.

b. La descripción de propuestas didácticas para la enseñanza del léxico en una metodología comunicativa para toda la etapa de secundaria.

7. La bibliografía en la que nos hemos basado para este estudio propone diversas estrategias para la didáctica del léxico:

a. Uso de los medios digitales.

b. Empleo de constelaciones.

c. Aplicación de técnicas de dramatización.

d. Aprendizaje por tareas.

8. Las propuestas didácticas realizadas están basadas en estas estrategias aunque somos conscientes de la necesidad de su puesta en práctica en el aula para garantizar su éxito.

\section{Referencias}

Giammatteo, M., Albano, H., Trombetta, A. M. y Ghío, A. (2001). Una propuesta de estrategias múltiples para el aprendizaje del léxico Español Actual, 76, 61-69. 
Gómez Molina, J. R. (1997). El léxico y su didáctica: una propuesta metodológica. Revista de Estudios de Adquisición de la Lengua Española (Reale), 7, 69-93.

Lázaro Carreter, F., Llupart, R., Mindán, J. y Gimeno, E. (2006). 4 secundaria. Lengua castellana y literatura. Madrid: Anaya.

Moreno, J. (2002). Propuestas para la enseñanza del vocabulario en el aula. Textos de Didáctica de la Lengua y de la Literatura, 31, 75-85.

Reyes Díaz, M. J. (1999). Descripción de la incorporación del léxico nuevo por los alumnos de primero de BUP. Revista de Estudios de Adquisición de la Lengua Española, 12, 85-92.

Rueda Bernao, M. J. (1994). Análisis de cuatro manuales para la enseñanza del español, lengua extranjera. Revista de Estudios de Adquisición de la Lengua Española, 2, 79-113.

Ruiz Zafón, C. (2004). La sombra del viento. Barcelona: Planeta.

Santiago, J. M. (2000). La selección del vocabulario didáctico: un nuevo enfoque. Revista de Lexicografía, 7, 155-172. 